\title{
DESENVOLVIMENTO DE UMA BANCADA ELÉTRICA UTILIZANDO ASSOCIAÇÃO DE RESISTORES.
}

Ivania Silva de Oliveira - ivania.oliveira@ufob.edu.br

Universidade Federal do Oeste da Bahia - Campus Multidisciplinar Bom Jesus da Lapa $(U F O B-C M B J L)$

47600000 - Bom Jesus da Lapa - Bahia

Christiane Karla Bispo da Costa - christiane.costa@ufob.edu.br

Universidade Federal do Oeste da Bahia - Campus Multidisciplinar Bom Jesus da Lapa $(U F O B-C M B J L)$

47600000 - Bom Jesus da Lapa - Bahia

Maiara Ferreira de Abreu - maiarabjllapa@hotmail.com

Universidade Federal do Oeste da Bahia - Campus Multidisciplinar Bom Jesus da Lapa $(U F O B-C M B J L)$

47600000 - Bom Jesus da Lapa - Bahia

Stefânia de Oliveira Silva - stefania.silva@ufob.edu.br

Universidade Federal do Oeste da Bahia - Campus Multidisciplinar Bom Jesus da Lapa $(U F O B-C M B J L)$

47600000 - Bom Jesus da Lapa - Bahia

Resumo: O presente trabalho apresenta o desenvolvimento de uma bancada didática de circuitos elétricos. O trabalho foi iniciado com o intuito de buscar uma solução que possa auxiliar os alunos no estudo de circuitos elétricos simples e seus componentes. Para isso, o trabalho tem como objetivo desenvolver uma bancada que simule os circuitos elétricos por meio do uso de peças individuais, cada uma com um componente elétrico com conexões padronizadas, possibilitando a combinação com quaisquer outros elementos da bancada. A construção da bancada elétrica utilizou conteúdos estudados em sala de aula, bem como lei de ohm, associação de resistores. As informações utilizadas no desenvolvimento do projeto, foram buscadas através de pesquisas bibliográficas e artigos, além do uso de alguns softwares. Além da descrição dos componentes, materiais e métodos utilizados para o desenvolvimento, o trabalho também apresenta alguns testes práticos. Após o término da construção da bancada, observou-se que, embora tenha funcionado de maneira correta, as lâmpadas não operavam com a sua luminosidade total, devido a utilização de uma fonte didática que fornecia uma corrente máxima de 3A. Acredita-se que os recursos aqui demonstrados, associados às aulas teóricas, propiciam um ambiente mais favorável à aprendizagem, fazendo com que o aluno tenha curiosidade e prazer em aprender, sendo uma valiosa ferramenta de complementação didática.

Palavras-chave: Circuitos elétricos. Aulas práticas. Bancada elétrica. 


\section{INTRODUÇÃO}

As atividades práticas em sala de aula têm ganhado cada vez mais importância nas instituições de ensino, com o objetivo de contribuir para o aprendizado dos alunos. A utilização de experimentos em aulas de circuitos elétricos consiste em uma ferramenta valiosa e complementar. Quando elaborados visando à aprendizagem do conteúdo, um experimento tem potencial para tornar-se uma eficaz ferramenta de conhecimento, apresentando grande capacidade para despertar o interesse dos alunos pelo conteúdo que se deseja ministrar, principalmente porque abordam esses conceitos dentro de um ambiente prático e lúdico. Quanto mais as experiências educacionais desenvolvidas pelos discentes se assemelharem às futuras situações, mais fácil torna o aprendizado em seus conhecimentos.

Neste sentido, buscou-se no presente trabalho uma forma de simular na prática um circuito elétrico, o qual pode ser definido como sendo um conjunto de componentes em que é possível verificar corrente elétrica (COTRIM, 2009). Pretende-se que o usuário possa perceber a relação entre teoria e prática ao relacionar as equações teóricas com os resultados práticos que podem variar devido às propriedades intrínsecas dos componentes usados. Propriedades essas que podem causar variações na condutibilidade elétrica dos materiais, variações de valores dos componentes elétricos, formas de medição, e demais fatores físicos, a fim de antever as influências e consequências dessas variações no resultado final.

O produto educacional proposto neste artigo busca utilizar aulas experimentais como ferramenta no processo de ensino e aprendizagem da engenharia na análise de circuitos elétricos. A proposta sugerida no presente trabalho utiliza não apenas aulas expositivas e laboratoriais e instrumentos de laboratório controlados, mas também as experiências mais simples, focando mais na busca de conceitos e análise de fenômenos por meio de sistemas de baixo custo.

O presente trabalho surgiu da tentativa de aumentar o interesse dos alunos pelos temas corrente elétrica, resistência e circuitos elétricos, simultaneamente a aprofundar a compreensão ao tornar os temas menos abstratos por meio da utilização de experimentos no tema em sala de aula, com referência às aulas práticas. A busca de informações para a construção da bancada e seus componentes foi em sua maior parte obtida por meio da pesquisa bibliográfica de obras relacionadas ao assunto, com ênfase no livro de Irwin (2013). O projeto deu-se como propósito a construção de uma bancada contendo lâmpadas de LED e lâmpadas fluorescentes, utilizando a teoria de circuitos elétricos, apresentado por estudantes de Engenharia Elétrica, na disciplina de Circuitos Elétricos I.

\section{FUNDAMENTAÇÃO TEÓRICA}

Ao analisar as dificuldades e os problemas enfrentados por estudantes do ensino médio na aprendizagem de certos conteúdos, verifica-se a necessidade em tornar o estudo mais concreto e mais próximo de sua realidade. Devido ao grau de abstração demandado no ensino de conceitos mais complexos em Eletricidade, habilidade ainda em fase de desenvolvimento nos alunos, a aplicação de experimentos em sala de aula, evidenciando a teoria em exemplos práticos que podem aparecer na rotina dos alunos, pode facilitar, motivar e incrementar o aprendizado destes conceitos entre os alunos, surgindo como uma ferramenta alternativa que vem a contribuir para uma melhoria desse processo de aprendizagem.

Segundo Gaspar (GASPAR; CASTRO,2005) é por meio dos experimentos que as ciências encantam e aguçam o interesse das pessoas. As atividades de demonstração não precisam se restringir às salas de aula, podendo ser apresentadas em outros ambientes, adequando a explicação dos temas a serem apresentados ao público à qual se destina. 
Os conceitos vistos em Eletricidade, em particular corrente elétrica, resistência elétrica e circuitos, costumam ser vistos pelos alunos como abstratos e distantes de seu cotidiano. A abordagem destes tópicos é frequentemente realizada por meio de aulas expositivas teóricas; entretanto, aulas experimentais podem complementar as aulas teóricas de maneira concreta e lúdica. Ao promover a experimentação em sala de aula, os alunos têm a oportunidade de verificar, testar e medir aqueles conceitos vistos apenas teoricamente, identificar associações de resistores e outros componentes em malhas, bem como simular malhas que representem uma instalação elétrica residencial.

A interação entre grandezas fundamentais é essencial para o ensino da Eletrodinâmica. A primeira lei de Ohm mostrou-se como conteúdo ideal para se perceber as proporcionalidades entre as amplitudes de cada grandeza envolvida, como também compreender como as cargas elétricas atuam no transporte de energia elétrica, desde a sua geração até a dissipação no receptor, conforme a potência requerida. Aplicando-se uma diferença de potencial $V$ a um condutor, fica estabelecida uma corrente elétrica de intensidade $i$. Considera-se, aplicado ao condutor, um mecanismo que permita a manutenção da temperatura constante. Alterando-se os valores da diferença de potencial $V$ e lendo-os com um voltímetro, pode-se também fazer leituras de $i$ usando um amperímetro.

Por essa definição, pode-se escrever a Equação da primeira lei de Ohm da seguinte forma:

$$
V=R i
$$

em que $R$ é a resistência.

Para Irwin (2013) os componentes de um circuito elétrico podem ser caracterizados de duas formas, pela tensão entre os terminais do componente ou pela corrente que passa por este componente. Estes componentes podem ser divididos em duas classes ativos ou passivos (IRWIN, 2013).

\subsection{Corrente Elétrica}

Cotrim (2009) define a corrente elétrica como sendo o movimento sistemático dos elétrons livres dentro do condutor, influenciado pela diferença de potencial ou fonte de tensão. Azevedo e Nunes (2012) afirmam que a corrente elétrica produz um campo magnético que varia dependendo do tipo de corrente, se a corrente for continua o campo magnético também será contínuo, se a corrente for alternada o campo magnético acompanhará a variação da corrente.

\subsection{Tensão}

A tensão em um circuito pode ser definida como a diferença de potencial entre dois polos diferentes (LUQUETA,1994), ou como a energia necessária para transportar a carga elétrica entre dois pontos (BURIAN JR.; LYRA, 2006). Essa diferença de potencial é conceituada por Gussow (1997) como sendo a capacidade de duas cargas diferentes realizarem trabalho. O mesmo autor também define que a diferença de potencial pode ser denominada como tensão elétrica.

\subsection{Resistores}

Para Irwin (2013), um componente de um circuito que ofereça resistência à passagem de corrente elétrica é chamado de resistor. Os resistores fazem parte dos elementos mais simples do circuito, pois não envolvem armazenamento de energia em campos. Para Luqueta (1994) as principais funções de um resistor em um circuito elétrico são a de atuar como um limitador 
de corrente ou como divisor de tensão. Portanto os resistores podem ser divididos em duas categorias, os fixos e variáveis (BURIAN JR.; LYRA, 2006).

Os resistores fixos possuem tensão e corrente proporcionais, este tipo de resistor possui dois terminais, os quais por sua vez são ligados nas extremidades do circuito resistivo, adicionando sua resistência total ao circuito. Portanto, um resistor fixo possui apenas um valor de resistência. Existem resistores que podem variar sua resistência dentro de um limite estabelecido pelo valor nominal, são denominados resistores variáveis, porém o termo mais usual empregado para denominá-los é como potenciômetro. Segundo Mileaf (1982) todo resistor em que se pode variar a resistência de modo fácil, e que resista a ajustes frequentes, podem ser definidos como resistores variáveis.

Os resistores variáveis não possuem faixas de cor para definição de seu valor como os resistores fixos, e sua leitura exata só poderá ser feita por meio do uso de algum equipamento de medição que possa fazer a leitura da resistência em ohms. Essa resistência encontrada mediante do equipamento de medição, em que ficará entre $0 \Omega$ e o valor nominal do resistor variável, gravado sobre o invólucro (MILEAF, 1982).

\subsection{Associação de Resistores}

Em um circuito muitas vezes é necessário fazer associações entre os resistores, seja para ter um funcionamento melhor ou para simplificar o circuito. E para tal feito utiliza-se associações quando necessário. Existem duas associações de resistores, série e paralelo. São considerados resistores conectados em série quando os mesmos são percorridos pela mesma corrente e podendo ser substituído por uma resistência equivalente. E resistores em paralelos, ocorrem quando a mesma tensão é comum a todos, contudo as correntes que passam por ele são diferentes.

De acordo com Irwin (2017) a resistência equivalente a N resistores em série e paralelo podem ser dadas pelas Equação (2) e (3), respectivamente:

$$
\begin{aligned}
& R_{S}=R_{1}+R_{2}+\cdots+R_{n} \\
& \frac{1}{R_{P}}=\frac{1}{R 1}+\frac{1}{R 2}+\cdots+\frac{1}{R n}
\end{aligned}
$$

\section{METODOLOGIA}

Para o desenvolvimento da bancada foram utilizados os seguintes procedimentos metodológicos:

- Coletar informações sobre os componentes elétricos utilizados na bancada por meio de pesquisas bibliográficas em livros e artigos, buscando informações específicas referentes a cada componente elétrico;

- Definir como e onde estes componentes elétricos serão fixados e conectados uns aos outros, com foco na praticidade sem interferir no funcionamento dos mesmos;

- Definir as medidas e materiais utilizados na bancada;

Para a construção da bancada utilizou-se os seguintes materiais:

- Madeira 0,5 m x 0,5 m;

- Interruptores;

- Caixas de luz;

- Lâmpadas de 24 V e 12 V; 


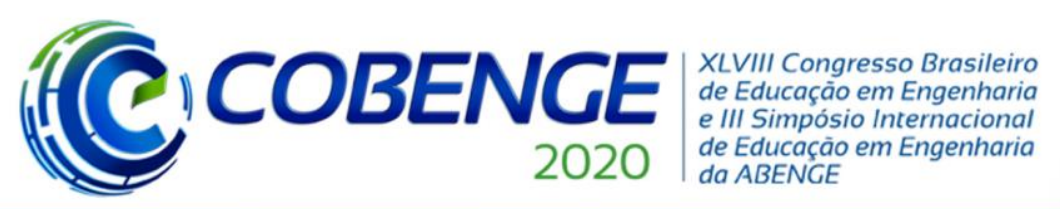

"Os desafios para formar hoje o engenheiro do amanhã"

- Fios para a instalação elétrica de 1,5 mm;

- Bocais para a lâmpada;

- Resistores de $15 \Omega$ com uma potência de $15 \mathrm{~W}$;

- Conector wago; Trilha de barramento; e

- Fonte contínua de $24 \mathrm{~V}$.

Durante a realização do projeto alguns obstáculos foram vencidos, no qual o primeiro relaciona-se a dificuldade de encontrar lâmpadas de $12 \mathrm{~V}$ incandescentes, conforme apresenta-se na Figura 1. Essas lâmpadas foram substituídas por lâmpadas de $24 \mathrm{~V}$.

Figura 1 - Circuito sugerido para a construção do projeto.

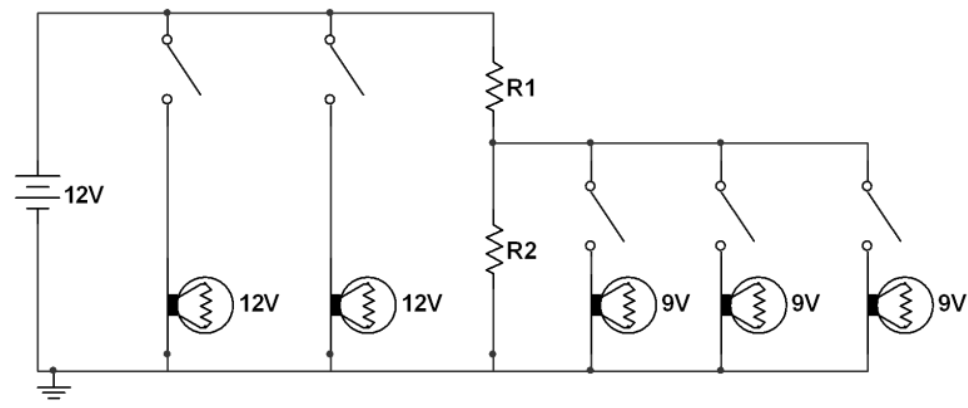

Fonte: Autoria Própria.

Para sancionar o problema, além da troca citada acima, utilizaram-se mais dois recursos, sendo um deles substituir a tensão da fonte de $12 \mathrm{~V}$ para $24 \mathrm{~V}$, pois assim poderia ser feito a utilização das lâmpadas. E a outra solução foi o uso de lâmpadas LED de $12 \mathrm{~V}$ com potência de $8 \mathrm{~W}$ em substituição as lâmpadas de $9 \mathrm{~V}$. Entretanto, para o emprego das lâmpadas de LED, algumas considerações foram postas, pois as mesmas possuíam resistências variáveis. Desta forma, tornou-se necessário uma nova análise matemática.

Encontrou-se os valores $\mathrm{R}_{1}$ e $\mathrm{R}_{2}$, conforme Figura 1, para que houvesse uma queda no valor da tensão. O primeiro passo foi o cálculo da resistência das lâmpadas de LED, por meio da Equação (4):

$$
P=R i^{2}
$$

onde $P$ é a potência, $R$ é a resistência e $i$ a corrente.

$\mathrm{Na}$ descrição do fabricante, a lâmpada de $8 \mathrm{~W}$ possui uma corrente nominal de $0,7 \mathrm{~A}$, assim:

$$
R=16,32 \Omega
$$

Como as lâmpadas LED encontram-se em paralelo, fazendo a associação de resistores utilizando a Equação (3), tem-se que a resistência equivalente vale:

$$
R_{e q}=5,44 \Omega
$$




\section{COBENGE (C) COBENCE 2020

"Os desafios para formar hoje o engenheiro do amanhã"

O circuito simplificado apresenta-se na Figura 2:

Figura 2- Circuito simplificado

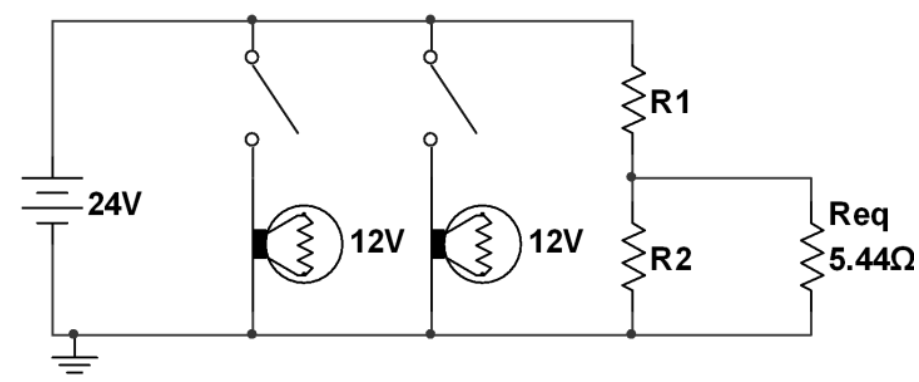

Fonte: Autores

Para o segundo passo calculou-se as resistências $R_{1}$ e $R_{2}$, analisando o circuito da Figura 3. Como $R_{2}$ está em paralelo com $R_{\text {eq }}$, qualquer valor que for alocado, resultará no mesmo valor de $R_{\text {eq.. }}$ Dada a resistência ser pequena, desconsiderou-se o valor inicial, no qual o circuito se posicionou com apenas uma resistência, conforme Figura 3:

Figura 3 - Circuito com apenas uma resistência.

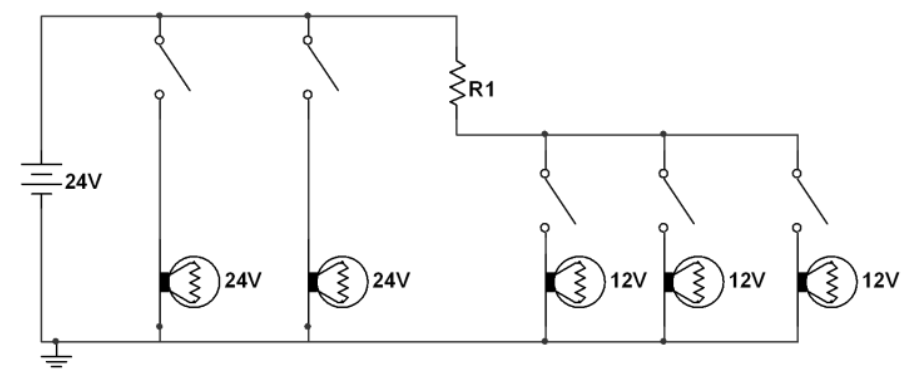

Fonte: Autoria Própria

Dada a corrente de cada lâmpada é de $0,7 \mathrm{~A}$ e se encontram em paralelo, a corrente total para alimenta-las conforme a Lei de Kirchhoff para correntes, é a soma da corrente das três lâmpadas, ou seja, 2,1A. Ao analisar a Figura 3, tem-se que a tensão que passa por $\mathrm{R}_{1}$, necessita de $12 \mathrm{~V}$ e a corrente de 2,1A, logo pela Lei de Ohm calculou-se a resistência de $\mathrm{R}_{1}$ :

$$
R_{1}=\frac{12}{2,1}=5,77 \Omega
$$

Como a potência nesse resistor é muito alta, torna-se necessário usar um resistor apropriado para suportar essa potência. Entretanto como não há um resistor com esse valor, associou-se três resistores de $15 \Omega$ em paralelo com o propósito de aproximar ao valor da requisitado. Após, os valores calculados, montou-se a bancada elétrica: 


\section{COBENCE de Educação em Engenharia

"Os desafios para formar hoje o engenheiro do amanhã"

Figura 4 - a) Desenho da bancada realizado no software Autodesk Inventor; b) Desenho da bancada 3D visão lateral; c) Desenho da bancada 3D visão frontal; d) bancada montada.

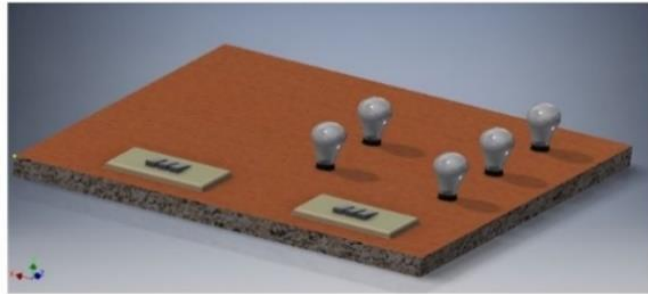

(a)

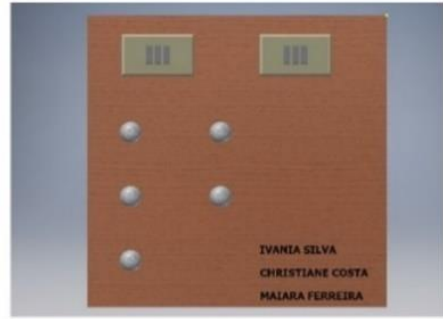

(c)

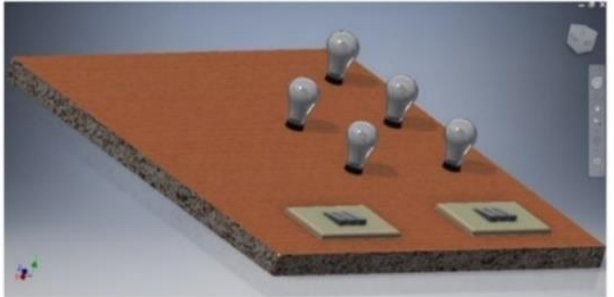

(b)

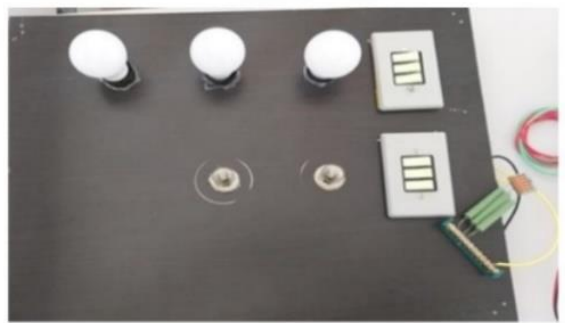

(d)

Fonte: Autoria Própria

\section{RESULTADOS E DISCUSSÕES}

Finalizada a construção da bancada, deu-se início aos testes de funcionamento. Ao acionar os interruptores para acender as lâmpadas de forma gradual, percebeu-se a redução da luminosidade, pois a fonte utilizada para alimentar o projeto não fornecia a quantidade necessária de corrente elétrica para que as lâmpadas operassem em sua total intensidade, sem interferir uma à outra. Na Figura 5 apresenta-se o processo gradual das lâmpadas.

Figura 5 - Lâmpadas sendo ligadas de forma gradual: (a) primeira lâmpada acessa; (b) segunda lâmpada acessa; (c) terceira lâmpada acessa; (d) quarta lâmpada acessa; (e) quinta lâmpada acessa.

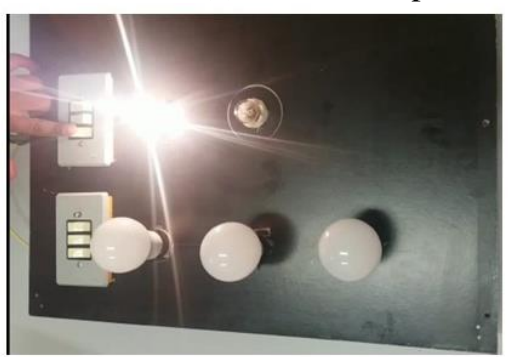

(a)

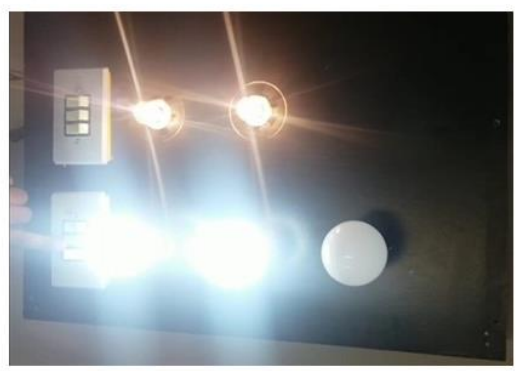

(d)

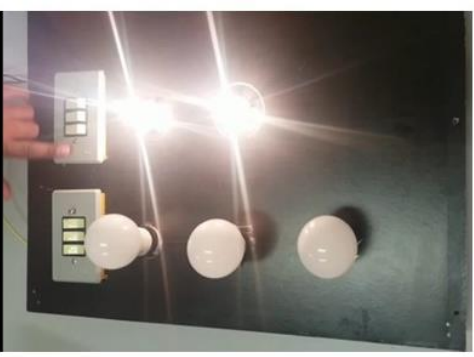

(b)
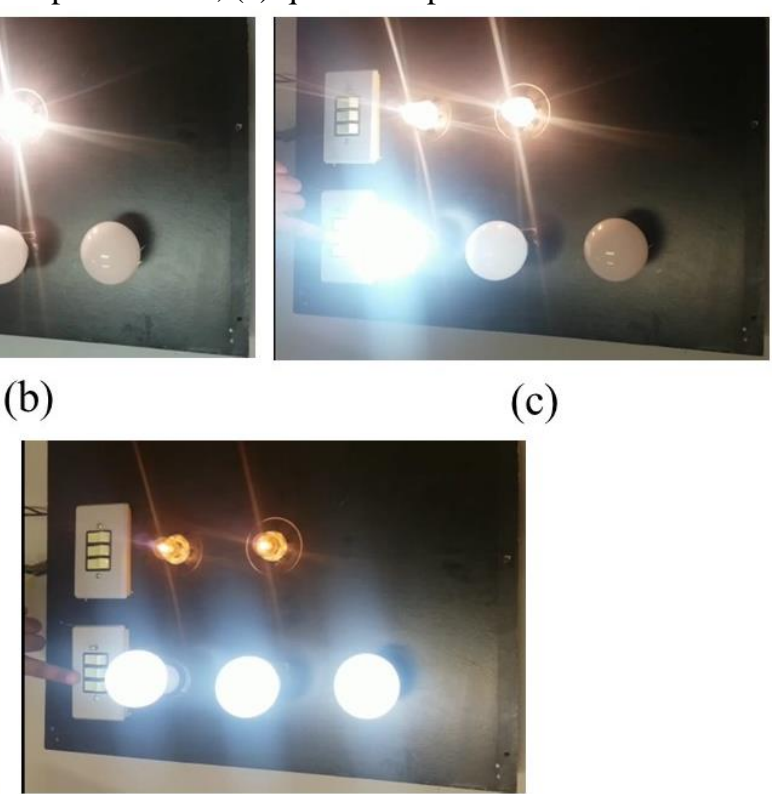

(e)

Fonte: Autoria própria 
Para que elas funcionassem da maneira correta, com grande intensidade em seu feixe de luz, seria necessário a alimentação com cerca de 4 A, pois as lâmpadas de LED individualmente possuem uma corrente nominal de $0,7 \mathrm{~A}$, e as incandescentes aproximadamente 0,9 A calculado a partir da sua potência. Como são três lâmpadas de LED em paralelo e duas incandescentes, ao somar as correntes resulta um valor próximo a 4 A. Entretanto, como a fonte utilizada é um equipamento didático, no qual fornece apenas uma corrente máxima de $3 \mathrm{~A}$, consequentemente diminui a eficiência da bancada.

\section{CONCLUSÃO}

Neste trabalho apresentou-se uma adaptação de uma bancada elétrica proposta pelo livro do Irwin que foi amplamente utilizado em sala de aula, sendo voltado para a realidade tanto na questão dos materiais e montagem, como na parte financeira.

A construção da bancada foi de grande impacto e acréscimo para a vida acadêmica dos estudantes, visto que foi possível visualizar que nem sempre a teoria presente nos livros se concilia com a prática, e é preciso se reinventar, principalmente tendo em vista que as discentes se encontram em uma universidade interiorizada, que teve como sua principal dificuldade a falta de materiais necessários para a construção da bancada. Com isso, foi preciso criatividade, paciência e determinação para resolução dos problemas que surgiram na construção da bancada, dando um novo olhar para uma parte da engenharia que até então as alunas não tinham destreza.

Durante a realização do projeto, foi possível observar que apesar dos obstáculos encontrados, o projeto foi concluído com precisão, criatividade e eficácia utilizando os conceitos adquiridos e propostos pela disciplina de Circuitos Elétricos I, disciplina esta, que se encontra no quarto semestre da grade curricular da universidade.

A única adversidade apresentada que não foi possível resolver de forma prática e que não escapasse do escopo do projeto, foi a falta de uma fonte elétrica que pudesse entregar uma corrente de $4 \mathrm{~A}$ para que as lâmpadas pudessem trabalhar no seu brilho mais intenso e pudessem funcionar como era esperado desde o início do projeto.

Visto isso, conclui-se que a construção desse tipo de projeto, torna-se benéfico, pois além de propiciar a prática do uso dos conhecimentos adquiridos em sala de aula, tem-se o manuseio de alguns softwares.

O presente trabalho foi apresentado tanto para os estudantes da disciplina, quanto para os estudantes do ensino médio da rede privada, ambos ficaram encantados com o projeto e a criatividade utilizada para a construção da bancada. Como estímulo para os alunos do ensino médio, ficou o desafio de construir outra bancada com materiais e equipamentos acessíveis e disponíveis a eles.

\section{Agradecimentos}

Primeiramente agradecemos à Deus e a nossa família, em segundo agradecemos aos professores Ricardo da Silva Reis e Leandro Machado Oliveira, pela ajuda prestada na construção da bancada tanto na parte teórica como na parte física, sobretudo na parte da instalação elétrica da bancada. Agradecemos também ao nosso colega de curso Márcio Antônio de Azevedo, pela ajuda na realização dos desenhos em formato 3D. 
"Os desafios para formar hoje o engenheiro do amanhã"

\section{REFERÊNCIAS}

ALEXANDER, Charles K.; SADIKU, Matthew N. Fundamentos de circuitos elétricos. $5^{\text {a }}$ edição, Porto Alegre: Editora AMGH. 2013.

AZEVEDO, E. R; NUNES, L. A. de O. Lei da Indução de Faraday. Disponível em: http://www.lla.if.sc.usp.br/ensino/Fisica3/11-LeideInducaodeFaraday.pdf. Acesso em: 12 maio. 2020.

COTRIM, Ademaro. Instalações elétricas. $5^{\text {a }}$ edição, São Paulo: Pearson Prentice Hall, 2009.

GASPAR, A., \& C., M. I. (2005). Atividades experimentais de demonstrações em sala de aula: Uma análise segundo o referencial da teoria de Vygotsky. UNESP-SP.

GUSSOW, Milton. Eletricidade Básica. Trad. Aracy Mendes da Costa. $2^{\text {a }}$ edição, São Paulo: Pearson Makron Books,1997.

IRWIN, J. David; NELMS, R Mark. Análise Básica de Circuitos para Engenharia. 10ª Edição, Rio de Janeiro: Livros Técnicos e Científicos, 2017.

LUQUETA, G. R. Curso básico de eletrônica analógica. Disponível em: http://www.netsoft.inf.br/aulas/4_EAC_Eletronica_Basica/7.3_Apostila_eletronica.pdf. Acesso em: 12 maio. 2020.

MILEAF, Harry. Eletricidade. $3^{\text {a }}$ Edição, São Paulo: Martins fontes, 1982.

\section{DEVELOPMENT OF AN ELECTRIC BENCH USING ASSOCIATION OF RESISTORS.}

Abstract: The present work presents the development of a didactic electric circuit board. The work was started with the aim of finding a solution that can help students in the study of simple electrical circuits and their components. To this end, the work aims to develop a bench that simulates the electrical circuits through the use of individual parts, each with an electrical component with standardized connections, allowing the combination with any other elements of the bench. The construction of the electric bench used contents studied in class, as well as ohm's law, association of resistors. The information used in the development of the project was sought through bibliographic research and articles, in addition to the use of some software. Besides the description of the components, materials and methods used for the development, the work also presents some practical tests. After the bench construction was finished, it was observed that, although it worked correctly, the lamps did not operate with their full brightness, due to the use of a didactic source that provided a maximum current of $3 A$. It is believed that the resources demonstrated here, associated with the theoretical classes, provide a more favorable environment for learning, making the student curious and enjoy learning, being a valuable tool for didactic complementation.

Keywords: Electrical circuits. Practical classes. Electric bench. 